\title{
Factors Affecting Fines Flocculation Performance with Cofactor-Polyethylene Oxide
}

\author{
M. R. Abdallah Qasaimeh ${ }^{1}$, Ahmad Qasaimeh ${ }^{2}$, F. Bani Hani ${ }^{1}$ \\ ${ }^{1}$ Chemical Engineering Department, AlHuson University College, AlBalqa Applied University, Irbid, Jordan \\ ${ }^{2}$ Department of Civil Engineering, Jerash University, Jerash, Jordan \\ Email: qasaimeh@hotmail.com, argg22@yahoo.com
}

Received December 25, 2013; revised January 7, 2014; accepted January 14, 2014

Copyright (C) 2014 M. R. Abdallah Qasaimeh et al. This is an open access article distributed under the Creative Commons Attribution License, which permits unrestricted use, distribution, and reproduction in any medium, provided the original work is properly cited. In accordance of the Creative Commons Attribution License all Copyrights (C) 2014 are reserved for SCIRP and the owner of the intellectual property M. R. Abdallah Qasaimeh et al. All Copyright (C) 2014 are guarded by law and by SCIRP as a guardian.

\begin{abstract}
In literature, neutral polyethylene oxide (PEO) flocculated fines at low shear rates, while with cofactor (CF) addition, the formed CF-PEO complex showed larger ability to bridge fines, producing flocs. In this work, some process factors were found having significant effects on fines flocculation. Increases in CF to PEO ratio at constant PEO enhanced the bridging bonds, causing increases in flocculation initial rate (efficiency), amplitude (floc size), and fastness (a decrease in characteristic time). On the other hand, an increase in stirring rate (shear rate) in flocculation vessel caused decreases in initial rate and amplitude, and an increase in the fastness. All runs showed transient flocculation; the amplitude increased with time, reached maximum at equilibrium, and then started to decrease showing deflocculation. In brief, the CF to PEO ratio and the shear rate were found important parameters in mill operation, having significant effects on flocculation efficiency, fastness, and floc size.
\end{abstract}

\section{KEYWORDS}

Polyethylene Oxide; Cofactor; Flocculation; Shearing and Bridging

\section{Introduction}

Flocculation process requires retention aid systems of dual- or multi-components to retain colloids and fines. The conventional cationic retention aids were found interfered with charged substances in furnishes, and the neutral and high molecular weight of polyethylene oxide (PEO) is used as an alternative [1,2]. PEO was found working at low shear rates [3] efficiently with a cofactor (CF) having phenol groups $[1,2,4]$. In flocculation and retention processes, the following cofactors: modified phenolic resin (MPR), sulphonated kraft lignin (SKL), tannic acid (TA), phenol formaldehyde resin (PFR) and sodium naphthalene sulphonate (SNS) were used in papermaking applications [5]. In literature, phenol cofactors CF and PEO are not adsorbed on some colloids, fines, and fibers, but their combinations were found effective [6]. Some hypothesis explained the work of retention aids; the most dominant one was the association-induce polymer bridging mechanism by van de Ven and Alince [7], who argued the mechanism of the net- work [6]. Inassociation-induce polymer bridging, the negative charge CF segments adsorbed on PEO coils expand and stiffen PEO coils to larger size $(\delta)$ due to repulsion among CF segments on the coils, making the coils capable to bridge surfaces. This large coil of PEO with CF is a CF-PEO complex, working as a polyelectrolyte capable to overcome the electrostatic double layer thickness $\left(\kappa^{-1}\right)$ with a thickness $\delta>\kappa^{-1}$ [8]. When PEO was used alone, the inducing polymer bridging (Figure 1(a)) showed that small PEO coil was not capable to bridge surfaces since $\delta>\kappa^{-1}$, but with $\mathrm{CF}$ addition (Figure 1(b)), the expanded CF-PEO complex $\left(\delta>\kappa^{-1}\right)$ bridged the surfaces [7,9].

Flocculation processes are either heteroflocculation of dissimilar particles or homoflocculation of similar ones. The rate of flocculation rate $r_{f}\left(=r_{\text {att }}-r_{\text {det }}\right)$ is a net of attachment rate $\left(r_{\text {att }}\right)$ and detachment or deflocculation rate $\left(r_{\text {det }}\right)[3,9,10]$. Re-flocculation rate $\left(r_{\text {ref }}\right)$ is the rate of the detached particles to form flocs again [11]. Attachment rate $r_{\text {att }}$ can be assumed here as comprising 


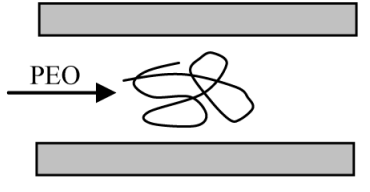

(a)

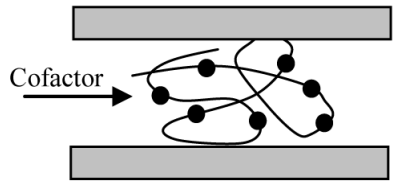

(b)
Figure 1. Schematic of association-induced polymer bridging. Here, (a) No chain adsorption onto surfaces; (b) Polymer adsorption and bridging occur when association stiffens the chain.

$r_{\text {ref }}$. One important parameter enhancing $r_{f}$ is flocculation efficiency $(\eta)$, or capture efficiency $\alpha(=\eta)$ as used for polymeric retention aids. When PEO is used with CF forming the CF-PEO complex in a suspension, the efficiency $\eta$ will be a function of the complex, or mainly of factors in PEO and CF. One factor is PEO quantity $(\Gamma)$ added to suspension to maintain the fractional coverage $(\theta)=\Gamma / \Gamma_{m}$ on the surfaces; here $\Gamma_{m}$ is the maximum quantity needed to maintain the full coverage $[9,12]$. The second factor is the CF quantity added, or the CF to PEO ratio $(\varphi)$ in suspension, which mainly determines the size $\delta$ of CF-PEO complex and the strength of the bridging bond between the complex and the surfaces $[3,11]$. The third factor is the shear rate $(G)$ subjected in flocculation vessel as a stirring rate $\mathrm{N}$ (rpm). In previous work [9], since the attachment rate applies $r_{a t t}=f\left(k_{a t t}, \eta\right)$, the shear rate $G$ that enhances the attachment rate constant $\left(k_{\text {att }}\right)$ and affects flocculation efficiency $\eta$, will also affect $r_{a t t}$. On the other hand, the shear rate $G$ that enhances the detachment rate constant $k_{\text {det }}(=f(G))$ will cause the detachment rate $r_{\text {det }}\left(=f\left(k_{\text {det }}\right)\right)$ to increase [9]. What's more, the shear rate $G$ effects on flocculation were reported in literature as a breakage of flocs that enhances $r_{\text {det }} \quad[9,13]$, and as a dissociation of the polyelectrolyte retention aid that causes $\eta$ to decrease [9]. Long exposure to hydrodynamic forces of similar intensity ought to be able to overcome the binding forces that hold flocs together [14]. The shear rate tends to break fiber flocs into one by eroding fiber from the surface of a floc $[15,16]$ or by splitting a floc into two [17-19]. Macromolecules can be described brittle; agitation to suspension of fiber flocculated by high mass polyelectrolyte tends to cause breakage, reducing the mean molecular mass [20-22]. Addition of cationic starch solution initially caused an extensive flocculation of fibers, which with continued agitation, the induced attachments between fibers were gradually broken resulting in a well dispersed suspension $[23,24]$. The forth factor is the number of parameters in PEO preparation, causing changes to PEO coil microstates prior to addition to flocculation process, and affecting flocculation efficiency $\eta$ after addition. These parameters are stirring intensity and time in PEO dissolu- tion unit; time in storage tank; dilution in preparation unit; and shear rate of a pump in transportation unit $[9,25]$. In literature, the first factor was expressed $\eta=2 \theta(1-\theta)$ $[9,12]$. The forth factors were parameters in preparation unit prior to PEO addition worked to dissociate the PEO entanglements decreasing their size $\delta$, causing a drop in flocculation efficiency $\eta[9,25]$. The PEO entanglements were found reformed after PEO granules dissolution in water resulted in a clear solution [26]. Two interacted coils are needed to build up a primary entanglement of a size larger than a single coil, while more coils will reform larger entanglements. In flocculation process, the PEO entanglement has a primary role: when PEO dosage is added as entanglements with $\mathrm{CF}$, the size of the resulted CF-PEO complex will get larger that increases the complex ability to bridge particles [9].

In this work, the second and the third factors become the main subject of this study, and the objectives are to determine the effects of the increases in the ratio $\varphi$ (at moderate values) and shear rate $G$ on flocculation efficiency $\eta$, amplitude (floc size), and fastness. To maintain this study, experiments of flocculation of fines were performed using CF-PEO retention aid at different shear rates $G$ and values of ratio $\varphi$. Since the detachment rate $r_{\text {det }}$ is considered negligible (zero) at initial time of flocculation, the initial rate of flocculation which becomes $r_{f}=r_{a t t}=f\left(k_{a t t}, \eta\right)$ can be taken as a measure of flocculation efficiency $\eta$ at constant shear rate $G$. The flocculation amplitude can be taken as a measure of floc size. The characteristic time of flocculation can be taken as a measure of flocculation fastness. Fines, the fiber fragments passing the 200 mesh pores of sizes less than $76 \mu \mathrm{m}$ [27], were used to determine flocculation characteristics with CF-PEO system in a wide range of particle size and a wide range of fines retention applications in papermaking and environment.

\section{Experimentation}

\subsection{Materials}

The fines used were separated by filtration of mixed pulps, taking the particles passed the 200 mesh $(76 \mu \mathrm{m})$ openings. Flocc 999; the PEO of 7 million molecular weight was used as a flocculent. Interac 1323 the phenolic material was used as a cofactor. Masson Maclaren Mill (Canada) provided the pulps, while I.Q.U.I.P Inc. (Canada) provided Flocc 999 and Intrac 1323.

\subsection{Experimental Setup}

The experimental setup used in this work (Figure 2) is the one used in previous work $[3,9,13]$. The fines $(0.1 \%$ consistency) was added to the beaker where mixed at constant stirring rate $\mathrm{N}$ (rpm), and then circulated by a peristaltic pump passing the photo cell of the Photome 


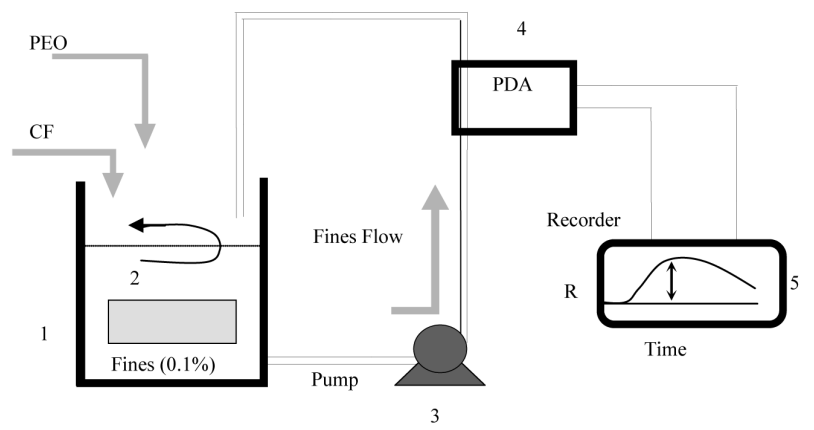

Figure 2. Experimental set up of flocculation vessel: 1 = flocculation vessel (Beaker), 2 = mixer, 3 = peristalic pump, $4=$ PDA, 5 = recorder.

tric Dispersion Analyzer (PDA) [28]. The PDA output signals, the direct current voltage $(D c)$ and the ratio $(R)$ of the alternating to direct current voltages, were plotted versus time by a recorder. After reaching steady state, CF was added to fines followed by PEO. The Dc signal, the voltage of the light transmitted the fines suspension, indicates particle concentration. The alternating voltage represents the root mean square (rms) of the transmitted light or the rms of the particle number, while ratio reading $R$ indicates particle size. The change of reading $R$ with time indicates the flocculation rate taken as a measure of flocculation intensity [28]. In this work, the vertical distance the pen moved in arbitrary unit (AU) was taken as $R$ reading. Both $D c$ and $R$ readings were plotted versus time $(t)$ by a recorder (Figure 3 ). The slope value of the curve $R$ at initial time gave initial rate of flocculation $r_{f}\left(=A_{m} / \tau\right)$, here, $A_{m}$ is flocculation amplitude at equilibrium, and $\tau$ is characteristic time of flocculation. Equilibrium time $\left(\tau_{e}\right)$ is the time the flocculation requires to reach equilibrium. Flocculation process was shown transient in all flocculation runs, deflocculating after reaching equilibrium. The slope the $R$ reading decreases at initial deflocculation is taken as initial rate of deflocculation $\left(r_{d}\right)$. The time required to reach zero amplitude at initial deflocculation rate is the characteristic time of deflocculation $\left(\tau_{d}\right)$.

\section{Results and Discussion}

In this work, two flocculation experiments were performed. One was to study the effect of CF to PEO ratio $\varphi$ at moderate values by adding different quantities of CF with constant PEO, keeping the other parameters constants. The second was to study the shear rate effect on flocculation intensity at different stirring rates $N$ (rpm), keeping other parameters constants. One result of experiment one (Figure 4) show that the flocculation intensity was enhanced when $\varphi$ was doubled; shown as an increase in the amplitude $A_{m}$ and initial rate $r_{f}$ which indicates an increase in floc size and flocculation efficiency $\eta$. Explanation of this increase is related to the increase in the CF segments adsorbed on PEO coils. Since the PEO coils are constant, doubling the CF quantity will double the ratio $\varphi$ which doubles the CF segments adsorbed on PEO coils. Here the PEO coils will be more stiffened and expanded resulting in larger CF-PEO complex that enhances flocculation efficiency $\eta$ and floc size. Furthermore, the number of the bridging bonds between the complex and fines surfaces will be doubled which strengthens the bridging bonds between PEO and surfaces $[3,8,9]$, enhancing flocculation efficiency and floc size. We should notice that we have used moderate values of $\varphi$, the one used in papermaking range to maintain the required specifications. The high values of $\varphi$, as reported [11] maintain small complexes and produce weak flocculation, are not in the scope of this work. The second result of experiment one was the transient flocculation of fines with CF-PEO system, recorded after equilibrium as a decrease in the ratio reading $R$ with time and estimated as a deflocculation rate $r_{d}$. This

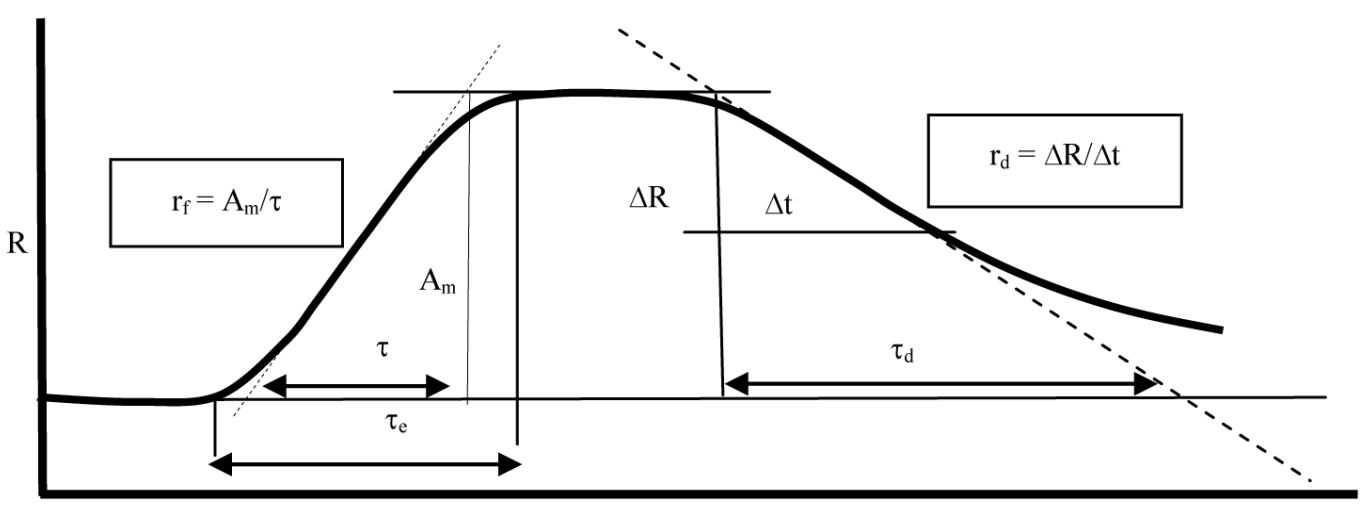

Time $\mathrm{t}$

Figure 3. Ratio reading $R$ is a measure of flocculation intensity with time. $r_{f}=$ initial rate of flocculation, $A_{m}=$ amplitude of flocculation at equilibrium, $\tau=$ characteristic time of flocculation, $\tau_{e}=$ equilibrium time of flocculation, $r_{d}=$ initial rate of deflocculation, $\tau_{d}=$ characteristic time of deflocculation. 
transient behavior was also reported in previous work [3] in flocculation processes with PEO alone enhanced by CF addition, where the transition behavior was ascribed to the instable PEO entanglement which dissociates with time. In this work, the deflocculation rate $r_{d}$ (Figure 4) is not only enhanced with CF addition, but shows a behavior with increases in $\varphi$. This increase in $\varphi$, the increase in CF segments adsorbed on PEO coils, will cause the repulsion intensity among PEO coils in CF-
PEO complex to increase. This increase in CF repulsion worked, in early flocculation, to expand the CF-PEO complex that enhanced flocculation efficiency $\eta$, and after flocculation reaching equilibrium, the CF repulsion in the formed flocs works to depart the PEO coils enhancing deflocculation.

Furthermore, the effect of ratio $\varphi$ increase on flocculation fastness (Figure 5) is indicated enhancing to the fastness, shown as a significant decrease in the characte-

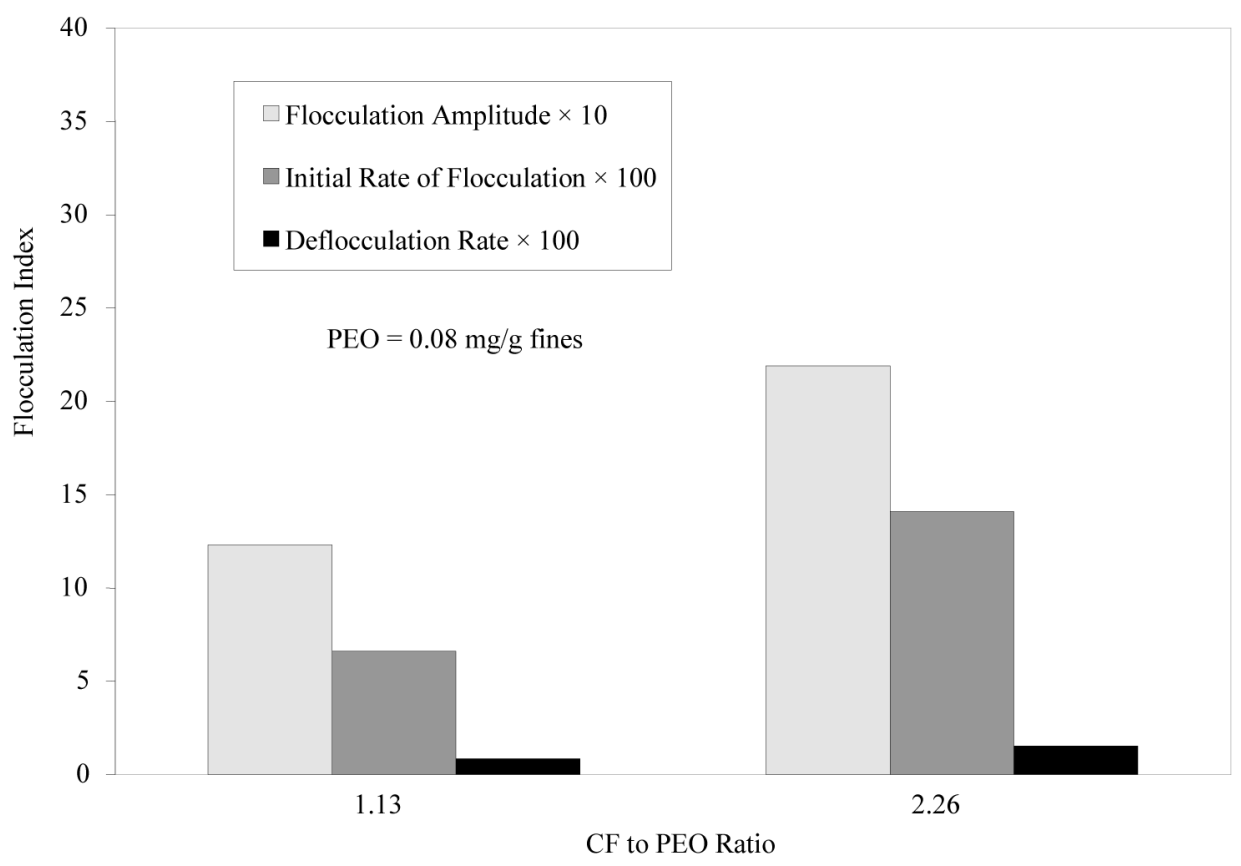

Figure 4. Effect of CF to PEO ratio ( $\phi$ ) on flocculation and deflocculation intensities.

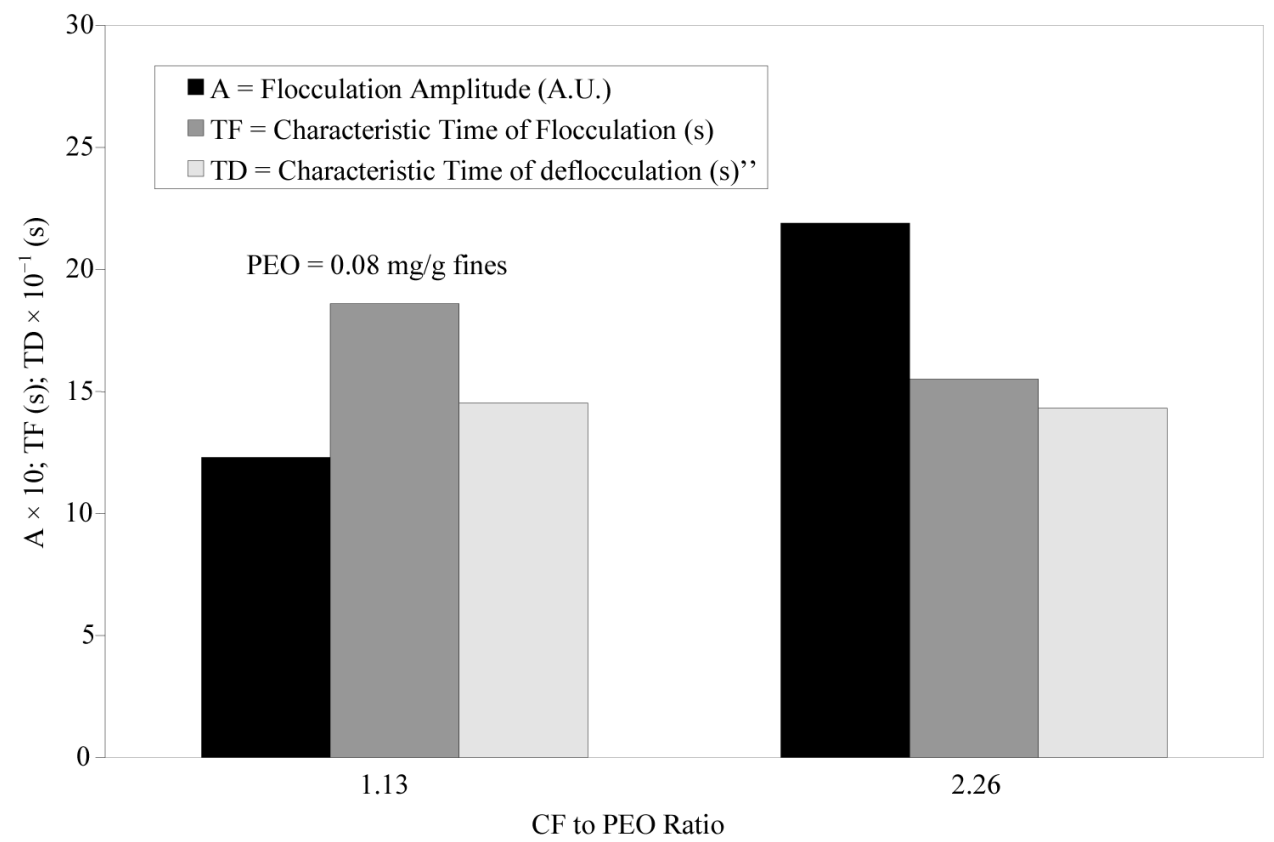

Figure 5. Effect of CF to PEO ratio ( $\phi$ ) on flocculation and deflocculation fastness. 
ristic time of flocculation $\tau$ with the increase in $\varphi$. The resulted characteristic time of deflocculation $\tau_{d}$ is shown about 7.8 to 9.2 times larger than the corresponding $\tau$, showing a relatively slow deflocculation, which allows sufficient time to save flocs prior to deflocculation. For more, the increase in ratio $\varphi$ (Figure 5) showed weak effect on deflocculation fastness, since $\tau_{d}$ was shown constant. The actual values of $\tau_{d}(s)$ at $\varphi$ $=1.13$ and 2.26 are respectively 145.5 and 143.3 . This small effect (deflocculation fastness is increased with the increase in $\varphi$ ) is expected significant at higher values of $\varphi$.

In experiment two, the effect of shear rate on flocculation intensity was studied, where $500 \mathrm{ml}$ of $0.1 \%$ fines were flocculated in a beaker at low and high stirring rates $N$ (rpm): 118, and 304. The circulated fines passed the PDA photocell via the transparent tubing by a peristaltic pump at low and constant velocity having a simple shear rate $\left(G_{t}\right)=287\left(\mathrm{~s}^{-1}\right)$. In each run, CF $(0.25 \mathrm{mg} / \mathrm{g}$ fines) was added and followed by PEO (0.12 mg/g fines) maintaining a constant and moderate ratio $\varphi \quad(=2.1)$. Results (Figure 6) show that the increase in stirring rate $N$ caused decreases in the amplitude $A_{m}$ and in initial rate of flocculation $r_{f}$, indicating decreases in floc size and flocculation efficiency $\eta$. In other hand, the increase in $N$ caused a decrease in the characteristic time of flocculation $\tau$, and relatively in equilibrium time $\tau_{e}$ since $\tau<\tau_{e}$ (Figure 3), shows faster flocculation.

Comparing the effect of the increases in shear rate $G$ and ratio $\varphi$ at moderate values on flocculation experiment, results have shown: the flocculation efficiency $\eta$ is increased with the increase in the ratio $\varphi$ and decreased with the increase in the shear rate $G$; the floc size is increased with the increase in the ratio $\varphi$ and decreased with the increase in the shear rate $G$; the flocculation fastness is increased with the increases in both ratio $\varphi$ and shear rate $G$. This result indicates that the bad selection of the shear rate $G$ in process will break large flocs resulted by CF, increasing operation cost. Furthermore, excess addition of CF at high $\varphi$ will produce fast flocculation with small flocs [11] and when $G$ is increased, the expected destructive effect of $G$ on flocs will be added. Furthermore, if floc is required of small size, the high shear rate $G$ with high dosage ratio $\varphi$ are seemingly not recommended, since the required size can be obtained by only increasing the shear rate $G$.

\section{Concluding Remarks}

In this work, increases in CF addition at constant PEO and in a moderate range of CF to PEO ratio caused increases in flocculation amplitude, rate and fastness, making these moderate ratios recommended for use in flocculation and retention processes. All flocculation runs with CF-PEO system were transient, showing deflocculation after equilibrium enhanced by CF to PEO ratio increase. Increases in the shear rate caused a decrease in flocculation amplitude producing small flocs, and a decrease in characteristic time of flocculation causing fast flocculation. Selection of a required floc size and flocculation fastness will be a matter of operation setting of CF to PEO value and shear rate.

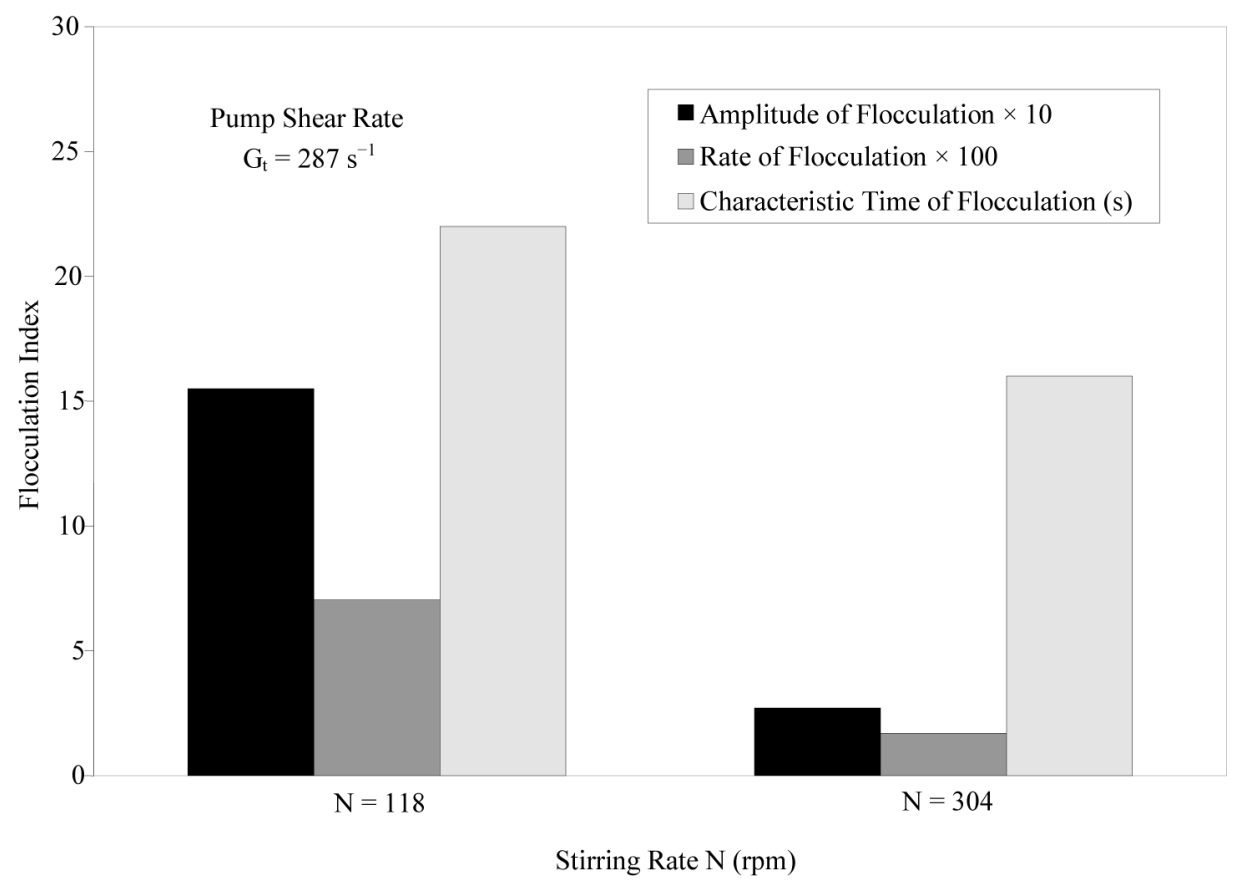

Figure 6. Effect of shear rate (stirring rate $\mathrm{N}$ ) on fines flocculation with CF-PEO in a beaker. 


\section{Acknowledgements}

1) I like to acknowledge Pulp and Paper Research Center/McGill, where these experiments were performed.

2) Dr. van de Ven T.G.M. and Dr. J. Paris for valuable discussions

3) Maclaren Masson Mill, and E.QU.I.P Inc. for material supply.

\section{REFERENCES}

[1] R. H. Pelton, L. H. Alen and H. M. Nugent, "Factors Affecting the Effectiveness of Some Retention Aids in Newsprint Pulp,” Srensk Pappestidn, Vol. 9, 1980, p. 251.

[2] R. H. Pelton, L. Allen and H. M. Nugent, "Novel Dual Polymer Retention Aids for Newsprint and Groundwood Specialties,” Tappi Journal, Vol. 64, No. 11, 1981, pp. 89-92.

[3] M. R. Abdallah Qasaimeh, "Causes of Transient Flocculation of Fines with Polyethylene Oxide and a Cofactor: Implications for Mill Performance," Colloids and Surfaces A: Physicochemical and Engineering Aspects, Vol. 386, No. 1-3, 2011, pp. 125-130.

http://dx.doi.org/10.1016/j.colsurfa.2011.07.008

[4] H. C. Tay and T. A. Canley, "Studies on Polyethylene Oxide as a Retention Aid in Wood Fiber Systems," Tappi Papermakers Conference, Vol. 1, 1982.

[5] T. G. M. van de Ven, "Mechanism of Fines and Filler Retention with PEO/Cofactor Dual Retention Aid Systems,” Journal of Pulp and Paper Science, Vol. 23, No. 9, 1997, pp. J447-J451.

[6] T. Lindström and G. Glads-Nordmark, "Flocculation of Latex and Cellulose Dispersion by Means of Transient Polymer Networks,” Colloids Surfaces, Vol. 8, No. 4, 1984, pp. 337-351. http://dx.doi.org/10.1016/0166-6622(84)80128-6

[7] T. G. M. van de Ven and B. Allince, "Association-Induced Polymer Bridging: New Insights into the Retention of Fillers with PEO,” Journal of Pulp and Paper Science, Vol. 22, No. 7, 1996, pp. J257-J263.

[8] J. A. De Witt and T. G. M. van de Ven, "The Effect of Neutral Polymer and Electrolyte on the Stability of Aqueous Polystyrene Latex," Advances in Colloid and Interface Science, Vol. 42, 1992, p. 41. http://dx.doi.org/10.1016/0001-8686(92)80019-T

[9] R. Mohammad Abdallah, "The Role of Entanglement in Polyethylene Oxide (PEO) Induced Flocculation of Fines," Ph.D. Thesis, Chemical Engineering Department, McGill University, Montreal, 2002.

[10] T. G. M. van de Ven, "Kinetic Aspects of Polymer and Polyelectrolyte Adsorption on Surfaces,” Advances in Colloid and Interface Science, Vol. 48, 1994, pp. 121-140. http://dx.doi.org/10.1016/0001-8686(94)80006-5

[11] M. R. Wu and T. G. M. van de Ven, "Flocculation and Reflocculation: Interplay between the Adsorption Behavior of the Components of a Dual Flocculant," Colloids and Surfaces A: Physicochemical and Engineering As- pects, Vol. 341, No. 1-3, 2009, pp. 40-45. http://dx.doi.org/10.1016/j.colsurfa.2009.03.034

[12] R. Hogg, "Collision Efficiency Factors for Polymer Flocculation,” Journal of Colloid and Interface Science, Vol. 102, No. 1, 1984, pp. 232-236. http://dx.doi.org/10.1016/0021-9797(84)90215-7

[13] M. R. Abdallah Qasaimeh, F. Bani Hani and A. M. Dawagreh, "Neutral Polyethylene Oxide with a Cofactor Recommended for Particle Flocculation,” Brazilian Journal of Chemical Engineering, Vol. 28, No. 3, 2011.

[14] M. Hubbe, "Flocculation of Cellulose Fibers," BioResources, Vol. 2, No. 2, 2007, pp. 296-331.

[15] T. W. Healy and V. K. La Mer, "The Energetics of Flocculation Redispersion of Polymers," Journal of Colloid Science, Vol. 19, No. 4, 1964, pp. 323-332. http://dx.doi.org/10.1016/0095-8522(64)90034-0

[16] T. P. Spicer and S. E. Pratsinis, "Sheared Induced Flocculation: The Evolution of Floc Structure and the Shape of the Size Distribution at Steady State,” Water Research, Vol. 30, No. 5, 1996, pp. 1049-1056. http://dx.doi.org/10.1016/0043-1354(95)00253-7

[17] A. N. Klomogorov, "On the Breakup of Droplets in Turbulent Flow,” Doklady Akademii Nauk SSSR, Vol. 66, No. 5, 1949, pp. 825-828.

[18] J. O. Hinze, "Fundamental of the Hydrodynamic Mechanism of Splitting in Dispersion Processes," AIChE Journal, Vol. 1, No. 3, 1955, pp. 289-295. http://dx.doi.org/10.1002/aic.690010303

[19] K. Higashitani, N. Inada and T. Ochi, "Floc Breakup in Contraction Flow to Orifice," AIChE Annual Meeting, San Francisco, 1989.

[20] M. D. Sikora and R. A. Startton, "The Shear Stability of Flocculated Colloids,” Tappi Journal, Vol. 64, No. 11, 1981, pp. 97-101.

[21] H. Tanaka, A. Swerin and L. Odberg, “Transfer of Cationic Retention Aid from Fibers to Fine Particles and Cleavage of Polymer Chains under Wet-End Papermaking Conditions,” Tappi Journal, Vol. 76, No. 5, 1993, pp. 157-163.

[22] S. Forsberg and G. Storm, "The Effect of Contact Time between Cationic Polymers and Furnish on Retention and Drainage,” Journal of Pulp and Paper Science, Vol. 20, No. 3, 1994, pp. J71-J76.

[23] J. C. Roberts, C. O. Au, G. A. Clay and C. Lough, "The Effect of $\mathrm{C}^{14}$-Labelled Cationic and Native Starches on Dry Strength and Formation,” Tappi Journal, Vol. 69, No. 10, 1986, pp. 88-93.

[24] J. C. Roberts, C. O. Au, G. A. Clay and C. Lough, "Study of the Effect of Cationic Starch on Dry Strength and Formation Using Carbon-14 Labelling,” Journal of Pulp and Paper Science, Vol. 13, No. 1, 1987, pp. J1-J5.

[25] T. G. M. van de Ven, M. Abdallah Qasaimeh, C. Pigeon and J. Paris, "PEO-Induced Flocculation of Fines: Effect of PEO Dissolution Conditions and Shear History," Colloids and Surfaces A: Physicochemical and Engineering Aspects, Vol. 248, 2004, pp. 151-156. http://dx.doi.org/10.1016/j.colsurfa.2004.09.010

[26] D. Kratochvil, B. Alince and T. G. M. van de Ven, "Floc- 
culation of Clay Particles with Poorly and Well Dissolved Polyethylene Oxide,” Journal of Pulp and Paper Science, Vol. 25, No. 9, 1999, pp. 331-335.

[27] J. P. Casey, "Pulp and Paper Chemistry and Chemical Technology,” 3rd Edition, Wiley Interscience, New York,
1979, pp. 1593-1607.

[28] J. Gregory, “Turbidity Fluctuations in Flowing Suspensions,” Journal of Colloid and Interface Science, Vol. 102, No. 1, 1984, pp. 232-236. 